\title{
Electron correlations in a partially filled first excited Landau level
}

\author{
Arkadiusz Wójs ${ }^{1,2}$ and John J. Quinn ${ }^{1}$ \\ ${ }^{1}$ Department of Physics, University of Tennessee, Knoxville, Tennessee 37996, USA \\ ${ }^{2}$ Institute of Physics, Wroclaw University of Technology, Wroclaw 50-370, Poland
}

\begin{abstract}
The form of electron correlations in a partially filled degenerate Landau level (LL) is related to the behavior of the anharmonic part of the interaction pseudopotential. Unlike in the lowest LL, the pseudopotential in the first excited LL is harmonic at short range. As a result, the incompressible states in this LL have different correlations, occur at different filling factors $\nu$, and cannot be described by a composite fermion model. The series of Laughlin-correlated states of electron pairs is proposed at $\nu=2+2 /\left(q_{2}+2\right)$ with integer $q_{2}$. It includes Moore-Read $\nu=\frac{5}{2}$ state and the $\nu=\frac{7}{3}$ state. Despite coincidence of the values of $\nu$, the latter state has different correlations than Laughlin state of single electrons at $\nu=\frac{1}{3}$ and, in finite systems, occurs at a different LL degeneracy (flux). PACS: 71.10.Pm, 73.43.Lp
\end{abstract}

Keywords: Fractional Quantum Hall Effect, Incompressible-Fluid State, Excited Landau Level

In the absence of another (kinetic) energy scale, correlations in a degenerate Landau level (LL) are completely determined by the form of electron-electron interaction [1]. Depending on the type of these correlations, the series of incompressible ground states (GS's) may occur at the specific values of the filling factor $\nu$, the elementary excitations of these GS's may have specific (quasiparticle) character and, consequently, the system (twodimensional electron gas, 2DEG, in a high magnetic field) may exhibit specific optical and transport properties.

For example, it turns out that the shortrange character of the Coulomb repulsion in the lowest $(n=0)$ LL makes the electrons maximally avoid those pair eigenstates with the smallest relative angular momenta $\mathcal{R}=1$, $3,5, \ldots$ 细. This tendency causes incompressibility at the specific values of the filling factor $\nu$, as well as the specific properties of the elementary excitations of these incompressible GS's [2,5,6]. The avoidance of pair states with small $\mathcal{R}$ can also be mimicked by a composite fermion (CF) transformation [7] in which the "hard core" at $\mathcal{R}<2 p+1$ is replaced by an attachment of $2 p$ vortices or magnetic flux quanta to each electron.
Correlations of this type do not generally occur in the excited LL's because of different behavior of the electron-electron repulsion. The condition necessary for Laughlin correlations is that the interaction pseudopotential [3], $V(\mathcal{R})$, is super-harmonic at short range [4, 8], and the Coulomb pseudopotential in the $n$th LL, $V_{\mathrm{C}}^{(n)}$, satisfies this condition only at $\mathcal{R} \geq 2 n+1$ [4]. 8 . Consequently, Laughlin correlations in the $n$th LL are not expected at $\nu>(2 n+2)^{-1}$, and neither will the $\mathrm{CF}$ picture be valid at these fillings. Therefore, it is not surprising that the half-filled state in the $n=1 \mathrm{LL}\left(\nu=\frac{5}{2}\right)$ is incompressible [9, 10], even though for $n=0$ all even-denominator fractions are compressible. It is less obvious that the correlations (and thus the reason for incompressibility) at $\nu=\frac{7}{3}$ and $\frac{8}{3}$ are different from those at $\nu=\frac{1}{3}$ and $\frac{2}{3}$, and that the CF model does not apply in the $n=1$ LL.

In this note, correlations in the $n=1 \mathrm{LL}$ are studied numerically. The energy spectra and the coefficients of fractional grandparentage (CFGP) [曰,11], $\mathcal{G}$, for the lowest energy states are calculated. The pair-correlation functions $\mathcal{G}(\mathcal{R})$ for the low-energy states are analyzed. The series of Laughlin-correlated states containing electron pairs is proposed. 


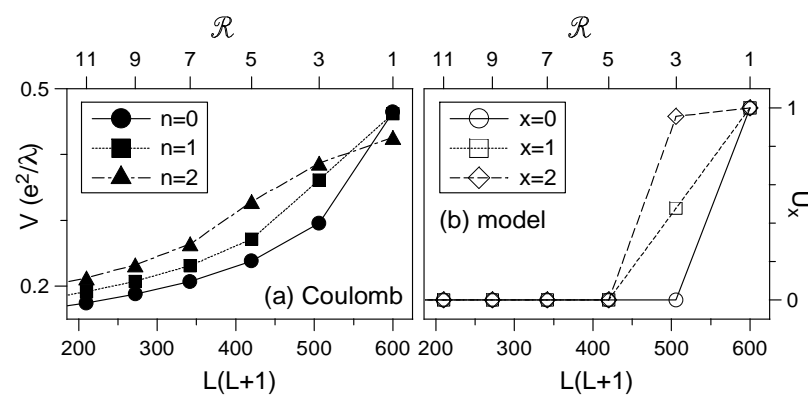

FIG. 1. Coulomb pseudopotentials in different LL's (a) and model pseudopotentials $U_{x}$ (b) calculated on Haldane sphere with $2 l=25$.

In our model [8], $N$ electrons are confined on a Haldane sphere [6], and the degeneracy of the $n$th LL, $g_{n}=2 l_{n}+1$, is controlled by the strength $2 S$ of the magnetic monopole inside the sphere $\left(l_{n}=S+n\right)$. The Coulomb matrix elements are calculated assuming zero width of the 2DEG, and the inter-LL scattering is neglected. All lengths and energies are given in the units of $\lambda$ (magnetic length) and $e^{2} / \lambda$. The many-body states are labeled by the length $(L)$ and projection $(M)$ of total angular momentum.

On a sphere, $\mathcal{R}=2 l-L$ and the harmonic

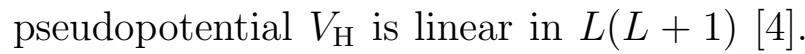
Only those pseudopotentials $V$ that decrease more quickly than $V_{\mathrm{H}}$ with increasing $\mathcal{R}$ cause Laughlin correlations 4,8 . 8 . It is clear from Fig. 目(a) that $V_{\mathrm{C}}^{(n)}$, the Coulomb pseudopotential in the $n$th LL, is super-harmonic in entire range of $\mathcal{R}$ only for $n=0$. To model different behavior of $V_{\mathrm{C}}^{(0)}$ and $V_{\mathrm{C}}^{(1)}$ at short range, a model pseudopotential shown in Fig. 1(b) can be used for which $U_{x}(1)=1, U_{x}(\mathcal{R} \geq 5)=0$, and $U_{x}(3)=x V_{\mathrm{H}}(3)$, where $V_{\mathrm{H}}(3)$ is the "harmonic" value such that $U_{1}$ is linear in $L(L+1)$ for $\mathcal{R}$ between 1 and 5 . While $U_{0}$ gives similar many-body energy spectra to $V_{\mathrm{C}}^{(0)}$, the (approximately) harmonic behavior of $V_{\mathrm{C}}^{(1)}$ at $\mathcal{R} \leq 5$ is well reproduced by $U_{1}$.

A few $n=1$ Coulomb energy spectra are compared with the spectra of $U_{1}$ in Fig. 2. The circles mark incompressible GS's in each

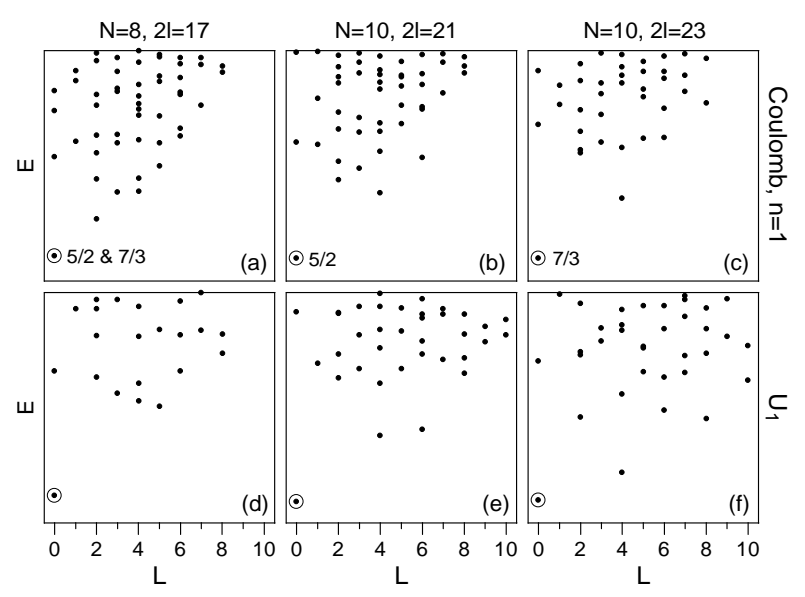

FIG. 2. The $N$-electron energy spectra calculated on Haldane sphere with different $2 l$ for Coulomb interaction in the $n=1 \mathrm{LL}$ (abc) and for model interaction $U_{1}$ (def).

frame, identified as the $\nu=\frac{5}{2}$ (Moore-Read [10]) and $\nu=\frac{7}{3}$ states. The similarity of the corresponding $V_{\mathrm{C}}^{(1)}$ and $U_{1}$ spectra (both very different from the $V_{\mathrm{C}}^{(0)}$ and $U_{0}$ spectra - not shown) confirms the fact that the essential feature of $V_{\mathrm{C}}^{(1)}$ that determines correlations in the $n=1$ LL is its harmonic behavior at short range.

Further confirmation of the essential role of this harmonicity comes from the comparison of CFGP profiles (pair-correlation functions) $\mathcal{G}(\mathcal{R})$ for the low-energy states, shown in Fig. 3. As an example, we display data for the lowest-energy $L=0$ states from the spectra of Fig. 2 and the (not shown) analogous spectra for $V_{\mathrm{C}}^{(0)}$ and $U_{0}$ (the latter correspond to different numbers of quasiholes, $\mathrm{QH}$, in Jain $\nu=\frac{2}{5}$ state). Clearly, the correlations obtained for the Coulomb interaction in the $n=0$ and 1 LL's are very different for both one-half and one-third filling, and they are very well reproduced by the model interactions $U_{0}$ and $U_{1}$, respectively. The main common feature of $\mathcal{G}(\mathcal{R})$ for $n=0$ is a strong minimum at $\mathcal{R}=1$ that can be viewed as a tendency for the electrons to maximally avoid this most strongly repulsive 


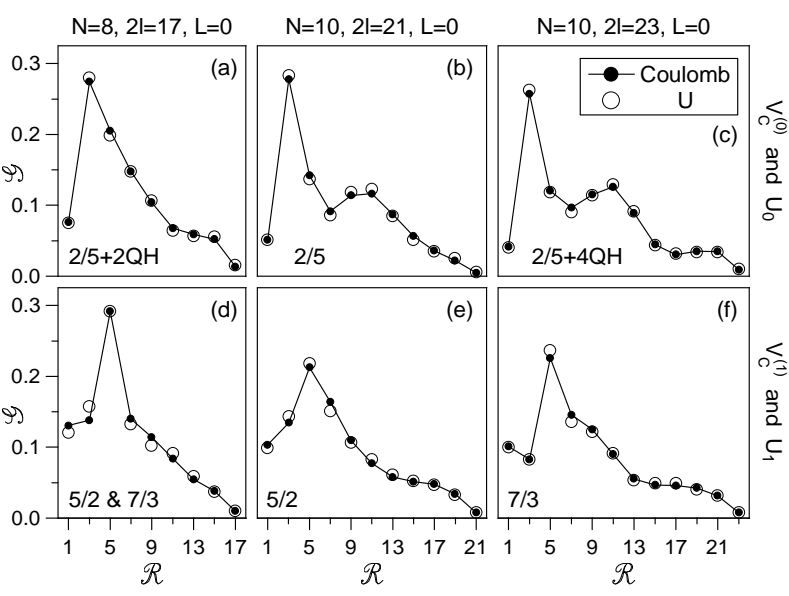

FIG. 3. CFGP profiles of the lowest-energy $N$-electron states at $L=0$, calculated on Haldane sphere with different $2 l$ : (abc) Coulomb interaction in the $n=0 \mathrm{LL}$ compared to model interaction $U_{0}$, and (def) Coulomb interaction in the $n=1 \mathrm{LL}$ compared to $U_{1}$.

pair state. Because of the sum rules satisfied by CFGP's 幽: (i) $\sum_{\mathcal{R}} \mathcal{G}(\mathcal{R})=1$ and (ii) $\frac{1}{2} N(N-1) \sum_{\mathcal{R}} L^{\prime}\left(L^{\prime}+1\right) \mathcal{G}(\mathcal{R})=L(L+1)+$ $N(N-2) l(l+1)$, where $L^{\prime}=2 l-\mathcal{R}$ and $L$ is the total $N$-electron angular momentum, the minimum at $\mathcal{R}=1$ causes maximum at $\mathcal{R}=3$. The harmonicity of $V_{\mathrm{C}}^{(1)}$ at $1 \leq \mathcal{R} \leq 5$ results in a different "prescription" for the CFGP profile that minimizes total interaction energy, $E=\frac{1}{2} N(N-1) \sum_{\mathcal{R}} V(\mathcal{R}) \mathcal{G}(\mathcal{R})$, in the $n=1$ LL. Namely, the total grandparentage from $\mathcal{R}=1$ and 3 is minimized, yielding a maximum at $\mathcal{R}=5$.

The optimum pseudopotential $U_{x}$ to model Coulomb correlations at $n=0$ or 1 can be found from a dependence of the leading CFGP's on $x$, shown in Fig. 4 for the same three many-body eigenstates of Fig. 3 . Clearly, the abrupt reconstruction of Laughlin correlations characteristic of $V_{\mathrm{C}}^{(0)}$ and $U_{0}$ occurs at $x \approx 1$, and the correlations resulting for $V_{\mathrm{C}}^{(1)}$ are best reproduced by $U_{x}$ with $x$ near this transition point. It is noteworthy that $\mathcal{G}(1) \approx \mathcal{G}(3)$ at $x \approx 1$, and that the total number of $\mathcal{R}=1$ pairs, $\frac{1}{2} N(N-1) \mathcal{G}(1)$, is

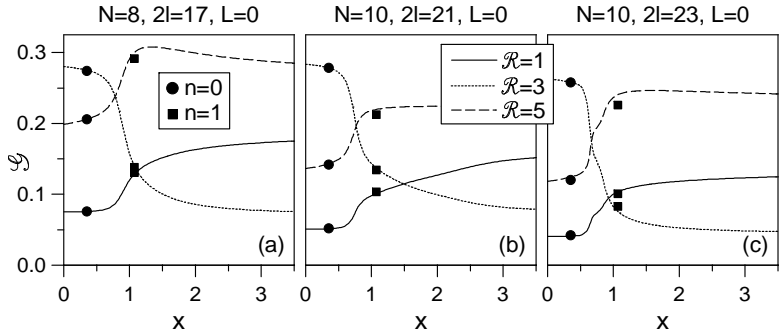

FIG. 4. Dependence of CFGP's $\mathcal{G}$ from pair states at $\mathcal{R}=1,3$, and 5 , on the anharmonicity parameter $x$ of the model pseudopotential $U_{x}$, calculated on Haldane sphere with different $2 l$ for the lowest-energy $N$-electron states at $L=0$. Symbols mark the values of $\mathcal{G}$ for the Coulomb pseudopotential in the $n=0$ and 1 LL's.

roughly equal to $\frac{1}{2} N$. This supports the idea of electron pairing in the $\nu=\frac{5}{2}$ state [10].

To identify the finite-size incompressible states in the $n=1 \mathrm{LL}$ (and to rule out the same character of the $\nu=\frac{1}{3}$ and $\frac{7}{3}$ states that coincidentally occur at the same filling of the $n=0$ and 1 LL's in the thermodynamic limit), in Fig. 5 we show the dependence of the excitation gap from the $L=0$ GS, $\Delta$, and of the leading CFGP's on $2 l$, calculated for $N=10$ and 12 . At $n=0$, large gaps $\Delta$ occur only at those $2 l$ corresponding to Laughlin or Jain GS's at $\nu=\frac{2}{3}, \frac{2}{5}, \frac{1}{3}$, etc., and coincide with the downward cusps in $\mathcal{G}(1)$ and upward peaks in $\mathcal{G}(3)$. At $n=1$, the gaps generally occur at different $2 l$ than at $n=0$ and coincide with the maxima of $\mathcal{G}(5)$. The horizontal lines labeled "5x2" and "6x2" show the values of $\mathcal{G}(1)=(N-1)^{-1}$ corresponding to the formation of $\frac{1}{2} N=5$ or 6 pairs with $\mathcal{R}=1$.

The facts that (i) $\mathcal{G}(1) \approx(N-1)^{-1}$ over certain range of $2 l$ for $n=1$ and (ii) Laughlin correlations keeping electrons maximally separated from one another no longer occur, suggest that electrons may indeed form $\mathcal{R}=1$ pairs in the $n=1 \mathrm{LL}$. Such pairs would then keep far apart from one another due to the super-harmonic behavior of $V_{\mathrm{C}}^{(1)}$ at larger $\mathcal{R}$. Laughlin pair-pair correlations can be for- 


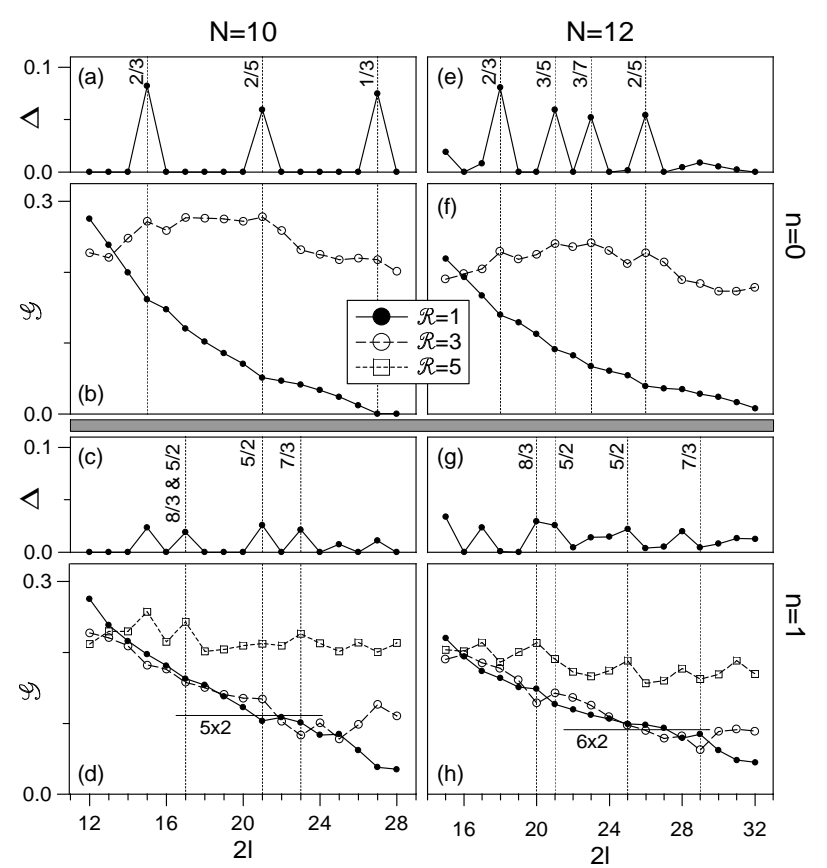

FIG. 5. Dependence of the excitation gap (aceg) and the leading CFGP's (bdfh) on $2 l$, calculated on Haldane sphere for $N=10$ (abcd) and 12 (efgh) electrons in the $n=0$ (abef) and $n=1$ (cdgh) LL.

mally introduced by a composite boson (CB) transformation applied to the (bosonic) pairs. The result is that incompressible Laughlin paired states are expected at the effective pair filling factors $\nu_{2}=\left(2 q_{2}\right)^{-1}$ with $q_{2}=1,2$, $3,4, \ldots$, that translate into the total electron filling factors of $\nu=2+2 /\left(q_{2}+2\right)=\frac{8}{3}$, $\frac{5}{2}, \frac{12}{5}, \frac{7}{3}, \ldots$ [8]. On Haldane sphere, these GS's and their particle-hole conjugates are expected at $2 l=\frac{1}{2} N\left(q_{2}+2\right)-\left(q_{2}+1\right)$ and $2 l=\frac{1}{2} N\left(q_{2}+2\right)+1$, respectively. Remarkably, the latter relation for $q_{2}=3$ is the same as for the Moore-Read (pfaffian) state [10], which therefore can be interpreted as a Laughlin $\nu_{2}=\frac{1}{6}$ state of $\mathcal{R}=1$ electron pairs.

The incompressible GS's for other values of $q_{2}$ have not been confirmed numerically. However, an $L=0$ GS occurs for any $N$ at $2 l=3 N-7$ (which gives $\nu=\frac{7}{3}$ in the thermodynamic limit, but is different from
$2 l=3 N-3$ of the Laughlin $\nu=\frac{1}{3}$ state) Also, its particle-hole conjugate $\nu=\frac{8}{3}$ state occurs in numerical spectra at any (even) $N$ and $2 l=\frac{3}{2} N+2$. Most likely, less than $\frac{1}{2} N$ pairs form in these states, and the Laughlin pair-pair and electron-pair correlations occur in such two-component plasma of $\mathcal{R}=1$ pairs and excess unpaired electrons (in analogy to the two-component Laughlin fluid of charged excitons and electrons [12]).

The authors acknowledge partial support of Grant DE-FG02-97ER45657 from Materials Science Program - Basic Energy Sciences of the US Dept. of Energy. AW acknowledges support of Polish KBN Grant 2P03B11118.

[1] R. E. Prange and S. M. Girvin, The Quantum Hall Effect, Springer-Verlag, New York (1987).

[2] R. Laughlin, Phys. Rev. Lett. 50, 1395 (1983).

[3] F. D. M. Haldane, in Ref. [1], chapter 8, p. 303; F. D. M. Haldane and E. H. Rezayi, Phys. Rev. Lett. 60, 956 (1988).

[4] A. Wójs and J. J. Quinn, Phil. Mag. B 80, 1405 (2000); J. J. Quinn and A. Wójs, J. Phys.: Cond. Mat. 12, R265 (2000).

[5] D. C. Tsui, H. L. Störmer, and A. C. Gossard, Phys. Rev. Lett. 48, 1559 (1982).

[6] F. D. M. Haldane, Phys. Rev. Lett. 51, 605 (1983); G. Fano, F. Ortolani, and E. Colombo, Phys. Rev. B 34, 2670 (1986).

[7] J. K. Jain, Phys. Rev. Lett. 63, 199 (1989).

[8] A. Wójs, Phys. Rev. B 63, 125312 (2001).

[9] R. L. Willet, J. P. Eisenstein, H. L. Störmer, D. C. Tsui, A. C. Gossard, and J. H. English, Phys. Rev. Lett. 59, 1776 (1987); J. P. Eisenstein, H. L. Störmer, L. Pfeiffer, and K. W. West, Phys. Rev. Lett. 62, 1540 (1989).

[10] G. Moore and N. Read, Nucl. Phys. B 360, 362 (1991); R. H. Morf, Phys. Rev. Lett. 80, 1505 (1998); E. H. Rezayi and F. D. M. Haldane, Phys. Rev. Lett. 84, 4685 (2000).

[11] A. de Shalit and I. Talmi, Nuclear Shell Theory, Academic Press, New York and London (1963).

[12] A. Wójs, I. Szlufarska, K. S. Yi, and J. J. Quinn, Phys. Rev. B 60, 11273 (1999). 